\title{
Quantitative Assessment of Breast Parenchymal Uptake on 18F-FDG PET/CT: Correlation with Age, Background Parenchymal Enhancement, and Amount of Fibroglandular Tissue on MRI
}

\author{
Doris Leithner ${ }^{1,2}$, Pascal A. Baltzer ${ }^{1}$, Heinrich F. Magometschnigg ${ }^{1}$, Georg J. Wengert ${ }^{1}$, Georgios Karanikas ${ }^{3}$, \\ Thomas H. Helbich ${ }^{1}$, Michael Weber ${ }^{1}$, Wolfgang Wadsak ${ }^{3}$, and Katja Pinker ${ }^{1}$ \\ ${ }^{I}$ Department of Biomedical Imaging and Image-Guided Therapy, Division of Molecular and Gender Imaging, Medical University of \\ Vienna, Vienna, Austria; ${ }^{2}$ Department of Diagnostic and Interventional Radiology, University Hospital Frankfurt, Frankfurt am Main, \\ Germany; and ${ }^{3}$ Department of Biomedical Imaging and Image-Guided Therapy, Division of Nuclear Medicine, Medical University of \\ Vienna, Vienna, Austria
}

Background parenchymal enhancement (BPE), and the amount of fibroglandular tissue (FGT) assessed with MRI have been implicated as sensitive imaging biomarkers for breast cancer. The purpose of this study was to quantitatively assess breast parenchymal uptake (BPU) on ${ }^{18} \mathrm{~F}-\mathrm{FDG}$ PET/CT as another valuable imaging biomarker and examine its correlation with BPE, FGT, and age. Methods: This study included 129 patients with suspected breast cancer and normal imaging findings in one breast (BI-RADS 1), whose cases were retrospectively analyzed. All patients underwent prone ${ }^{18} \mathrm{~F}-\mathrm{FDG} \mathrm{PET} / \mathrm{CT}$ and 3-T contrast-enhanced MRI of the breast. In all patients, interpreter 1 assessed BPU quantitatively using SUV $\mathrm{Smax}_{\text {. Interpreters }}$ 1 and 2 assessed amount of FGT and BPE in the normal contralateral breast by subjective visual estimation, as recommended by BI-RADS. Interpreter 1 reassessed all cases and repeated the BPU measurements. Statistical tests were used to assess correlations between BPU, BPE, FGT, and age, as well as inter- and intrainterpreter agreement. Results: BPU on ${ }^{18} \mathrm{~F}-\mathrm{FDG}$ PET/CT varied among patients. The mean BPU SUV $V_{\max } \pm$ SD was $1.57 \pm 0.6$ for patients with minimal BPE, $1.93 \pm 0.6$ for mild BPE, $2.42 \pm 0.5$ for moderate BPE, and $1.45 \pm$ 0.3 for marked BPE. There were significant $(P<0.001)$ moderate to strong correlations among BPU, BPE, and FGT. BPU directly correlated with both BPE and FGT on MRI. Patient age showed a moderate to strong indirect correlation with all 3 imaging-derived tissue biomarkers. The coefficient of variation for quantitative BPU measurements with $S U V_{\max }$ was $5.6 \%$, indicating a high reproducibility. Interinterpreter and intrainterpreter agreement for BPE and FGT was almost perfect, with a $\mathrm{k}$-value of 0.860 and 0.822 , respectively. Conclusion: The results of our study demonstrate that BPU varied among patients. BPU directly correlated with both BPE and FGT on MRI, and BPU measurements were highly reproducible. Patient age showed a strong inverse correlation with all 3 imaging-derived tissue biomarkers. These findings indicate that BPU may serve as a sensitive imaging biomarker for breast cancer prediction, prognosis, and risk assessment.

Received Feb. 29, 2016; revision accepted Apr. 12, 2016.

For correspondence or reprints contact: Thomas H. Helbich, Medical University of Vienna/Vienna General Hospital, Waehringer Guertel 18-20, 1090 Vienna, Austria.

E-mail: thomas.helbich@meduniwien.ac.at

Published online May 26, 2016.

COPYRIGHT (c) 2016 by the Society of Nuclear Medicine and Molecular Imaging, Inc.
Key Words: ${ }^{18} \mathrm{~F}-\mathrm{FDG}$ PET/CT; breast parenchymal uptake; magnetic resonance imaging; imaging biomarker

J Nucl Med 2016; 57:1518-1522

DOI: 10.2967/jnumed.116.174904

\section{A}

ccurate risk assessment is pivotal for women at high risk for breast cancer to personalize risk-reduction interventions (1). In clinical practice, several risk-reduction strategies are being used, ranging from lifestyle changes and chemoprevention to invasive interventions such as prophylactic mastectomy and salpingo-oophorectomy (2-6). Although these interventions are efficient in reducing cancer risk $(6,7)$, they are associated with substantial side effects $(8,9)$ and can negatively affect the quality of life (10). However, it currently remains uncertain which level of intervention is indicated for individual women (11), leading to potential overtreatment and undertreatment. Therefore, methods are urgently needed to better determine the likelihood of response to risk-reduction interventions and thus better guide risk management decisions.

MRI of the breast allows a 3-dimensional assessment of both the anatomy and the physiologic activity of breast tissue. In addition, MRI provides insight about the amount of fibroglandular tissue (FGT), as well as background parenchymal enhancement (BPE). Thus, FGT and BPE are considered more sensitive predictive and prognostic imaging biomarkers than density assessment with mammography (12-18).

Similar to MRI, PET using the radiotracer ${ }^{18} \mathrm{~F}-\mathrm{FDG}$ provides insight into the physiologic activity of the normal breast parenchyma through the depiction of tissue glucose metabolism (1924). This ${ }^{18} \mathrm{~F}-\mathrm{FDG}$ uptake is defined as breast parenchymal uptake (BPU). Thus, it could be that BPU might also serve as an important imaging biomarker in breast cancer.

With the worldwide implementation of hybrid PET/MRI systems and dedicated breast PET systems, there is the potential to simultaneously assess and monitor these imaging biomarkers of breast cancer $(19,25,26)$. To define the value of BPU, BPE, and FGT as imaging biomarkers for breast cancer and use them for better guidance of risk management decisions, information about their correlations and 
reproducibility is needed. However, to our knowledge, no such information currently exists. Therefore, the aim of this study was to quantitatively assess BPU on ${ }^{18} \mathrm{~F}$-FDG PET/CT and examine its correlation with age and with BPE and FGT on MRI.

\section{MATERIALS AND METHODS}

The institutional review board approved this prospective, singleinstitution study and retrospective data analysis, and all patients gave written informed consent.

\section{Patients}

From December 2009 to April 2014, 172 consecutive patients with suspected breast cancer were evaluated for inclusion in this study. To be included, the patients had to be at least $18 \mathrm{y}$ old, not pregnant, and not breastfeeding; had to have a suggestive finding on mammography or breast ultrasonography (i.e., asymmetric density, architectural distortion, breast mass, or microcalcifications [BI-RADS 0 , further imaging warranted; BI-RADS 4, suspicious abnormality; or BI-RADS 5, highly suggestive of malignancy]) that had not been previously treated; and could have no contraindications to MRI or MRI contrast agents. Twenty-eight patients had to be excluded because of incomplete examinations or prior treatment. The remaining 144 patients underwent ${ }^{18} \mathrm{~F}$-FDG PET/CT and MRI. In 30 patients, the imaging revealed an abnormality in the contralateral breast (BI-RADS 2-5). All suggestive lesions were histopathologically verified by either image-guided or surgical biopsy. Fifteen of these patients were excluded because of an MRI or PET imaging finding in the contralateral breast; that is, not the breast in which there had been an imaging abnormality on mammography or breast ultrasonography. The remaining 129 patients (mean age $\pm \mathrm{SD}, 57.9 \pm 3.9$ y; range, $18-87$ y) were evaluated in the retrospective analysis.

Several patients examined in this study were previously analyzed in a different context $(19,22)$.

\section{Imaging}

The ${ }^{18} \mathrm{~F}$-FDG PET/CT and MRI examinations were performed no more than $6 \mathrm{~d}$ apart (mean, $2.26 \mathrm{~d}$; range, 0-6 d; same day, $n=57$; $1 \mathrm{~d}, n=32 ; 2 \mathrm{~d}, n=11 ; 3 \mathrm{~d}, n=9 ; 4 \mathrm{~d}, n=12 ; 5 \mathrm{~d}, n=5 ; 6 \mathrm{~d}, n=1)$.

A prone PET/CT dataset over the breasts was acquired using a combined PET/CT in-line system (Biograph 64 TruePoint; Siemens), allowing the same patient geometry as with MRI. The whole-body PET/CT system is equipped with a high-resolution PET scanner and a 64-detector CT scanner. The patients fasted $6 \mathrm{~h}$ before injection of approximately $300 \mathrm{MBq}$ of ${ }^{18} \mathrm{~F}-\mathrm{FDG}$, depending on patient weight. Blood glucose levels were less than $150 \mathrm{mg} / \mathrm{dL}(8.3 \mathrm{mmol} / \mathrm{L})$ at the time of tracer application. Uptake time was $60 \mathrm{~min}$. No contrast agent was injected for the CT scan, which was used only for attenuation correction. PET images were reconstructed using the TrueX algorithm (Siemens) (22).

MR imaging was performed with the patient prone using a 3-T Tim Trio scanner (Siemens) and a 4-channel breast coil (InVivo). In premenopausal patients, MRI was performed between the seventh and 14th days of the menstrual cycle to minimize hormonal influence on BPE (27).

The MRI protocol consisted of 3 sequences. The first was a T2weighted turbo spin echo sequence with fat suppression (repetition time/ echo time, 4,800/9 ms; field of view, $340 \mathrm{~mm}$; 48 superior-inferior slices $3 \mathrm{~mm}$ thick; flip angle, $128^{\circ}$; matrix, $384 \times 512$; acquisition time, $2 \min 16 \mathrm{~s})$. The second and third were a split-dynamics, contrastenhanced T1-weighted volume-interpolated breath-hold examination (repetition time/echo time, 3.62/1.4 ms; field of view, $320 \mathrm{~mm} ; 72$ slices; 1.7-mm isotropic spatial resolution; matrix, $192 \times 192$; one average; $13.2 \mathrm{~s}$ per volume) and a T1-weighted turbo fast low-angle shot 3-dimensional sequence with selective water-excitation (repeti- tion time/echo time, 877/3.82 ms; field of view, $320 \mathrm{~mm}$; 96 slices; 1 $\mathrm{mm}$ isotropic spatial resolution; matrix, $320 \times 134$; one average; $2 \mathrm{~min}$ ) with a total acquisition time of $15 \mathrm{~min} 20 \mathrm{~s}$.

A standard dose $(0.1 \mathrm{mmol} / \mathrm{kg}$ of body weight $)$ of gadoterate meglumine was administered intravenously as a bolus using a power injector (Spectris Solaris EP; Medrad) at $4 \mathrm{~mL} / \mathrm{s}$ with a saline flush after injection. The total MRI examination time was approximately $18 \mathrm{~min}$.

\section{Data Analysis}

${ }^{18} \mathrm{~F}$-FDG PET/CT. After reconstruction of the ${ }^{18} \mathrm{~F}-\mathrm{FDG}$ PET/CT images, a 3-dimensional region of interest was carefully drawn on the contralateral normal breast around the glandular breast tissue, as determined by visual inspection of the subsequent 4-6 PET/CT scan slices by a breast radiologist under the supervision of a nuclear medicine physician. The nipple and areola area were excluded from regionof-interest placement. From these regions of interest, the $\mathrm{SUV}_{\max }$ was calculated. In all patients, BPU SUV $\mathrm{Sax}_{\text {max }}$ measurements were repeated by interpreter 1 to assess reproducibility.

MRI. FGT and BPE of the normal contralateral breast were qualitatively assessed on the MR images by 2 independent interpreters using the revised American College of Radiology (ACR) BI-RADS classification (28). For calculation of intrainterpreter agreement, interpreter 1 reassessed all cases. As recommended by BI-RADS, FGT and BPE were assessed by visual subjective estimation for each MRI study. FGT was classified as ACR A if the breasts were almost entirely fatty, ACR B if scattered FGT was present, ACR C if there was heterogeneous FGT, and as ACR D if there was extreme FGT content. BPE was graded as minimal, mild, moderate, or marked on the basis of contrast-enhanced images approximately $90 \mathrm{~s}$ after contrast medium injection.

\section{Statistical Analysis}

Statistical analysis was performed using SPSS Statistics, version 22.0 (IBM). Metric data, such as BPU SUV $\mathrm{SUx}_{\text {max }}$ and age, are described using mean $\pm \mathrm{SD}$, and nominal and ordinal data are presented using absolute frequencies and percentages. To compare average age and BPU for different BPE and FGT grades, 1-way analyses of variance and post hoc Tukey range tests were performed. The Pearson correlation coefficient $(r)$ was used to assess for correlations between age and BPU, and Spearman rank correlation $(\rho)$ was used to assess for correlations between BPE and FGT. To calculate inter- and intrainterpreter agreement, the Cohen $\kappa$ was used for FGT and BPE and the coefficient of variance was used for BPU. A $P$ value of 0.05 or less was considered statistically significant. A correlation coefficient matrix was prepared showing numeric, color-coded, and size-coded Pearson correlation coefficients for BPU SUV max $_{\text {ax }}$ on ${ }^{18} \mathrm{~F}-\mathrm{FDG}$ PET/CT, BPE and FGT on MRI, and age for both interpreters, as well as reassessment of BPU SUV max $_{\text {ax }}$ by interpreter 1 , with 0 indicating no correlation, $0.1-0.2$ a weak correlation, $0.3-0.4$ a moderate correlation, $0.5-0.7$ a strong correlation, and 0.8 or higher an excellent correlation (Fig.1).

\section{RESULTS}

Mean, SD, minimum, and maximum BPU SUV $\max$ on ${ }^{18} \mathrm{~F}-\mathrm{FDG}$ PET/CT were $1.81,0.61,0.88$, and 4.55 , respectively. The mean

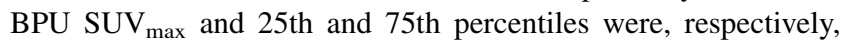
$1.57 \pm 0.6$ and $1.27-1.88$ for minimal BPE, $1.93 \pm 0.6$ and $1.44-$ 2.31 for mild BPE, $2.42 \pm 0.5$ and 2.06-2.85 for moderate BPE, and $1.45 \pm 0.3$ and 1.09 for marked BPE. Thus, BPU on ${ }^{18} \mathrm{~F}-\mathrm{FDG}$ PET/CT varied among patients.

BPE was minimal in 58 patients $(44.96 \%)$, mild in $54(41.86 \%)$, moderate in $14(10.85 \%)$, and marked in $3(2.33 \%)$. FGT was classified as ACR A in 33 patients, ACR B in 59, ACR C in 27, and ACR D in 10. The mean BPU SUV $\max$ and 25th and 75th 


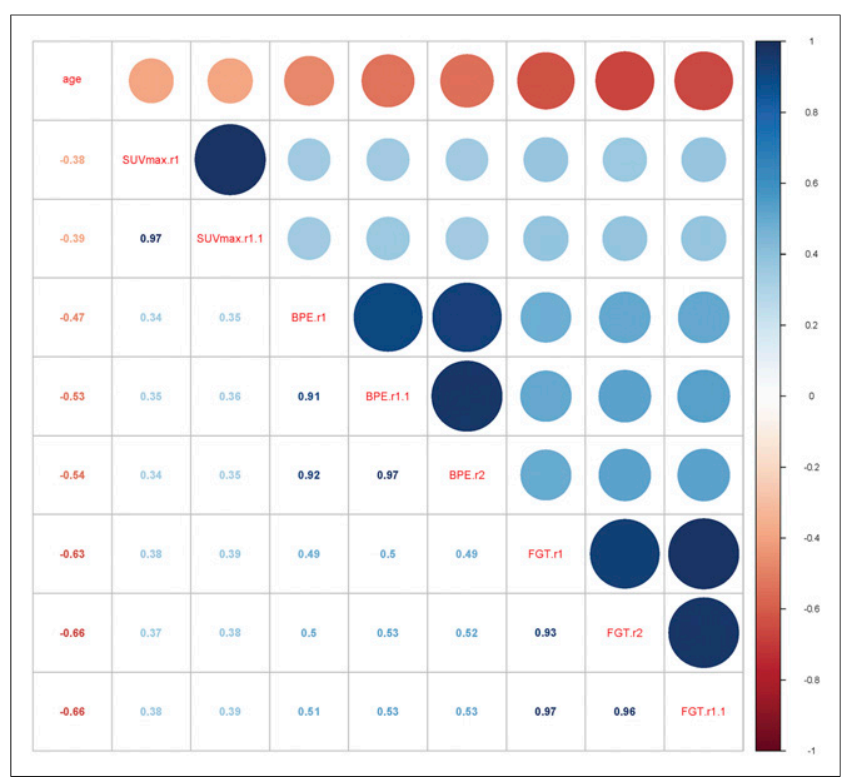

FIGURE 1. Matrix showing numeric, color-coded, and size-coded Spearman rank correlation coefficients for BPU SUV ${ }_{\max }$ on ${ }^{18} \mathrm{~F}-\mathrm{FDG}$ $\mathrm{PET} / \mathrm{CT}$, BPE, FGT, and age for both interpreters and reassessment of BPU SUV max $_{\max }$ by interpreter 1. Correlations ranged from moderate to strong and are marked in blue if positive and red if negative.

percentiles were, respectively, $1.54 \pm 0.5$ and 1.15-1.89 for ACR A, $1.75 \pm 0.5$ and 1.36-2.16 for ACR B, $2.01 \pm 0.75$ and 1.482.44 for ACR C, and $2.29 \pm 0.66$ and 1.79-2.54 for ACR D. Detailed ACR classification results for BPE and FGT for both interpreters and interpretation rounds are summarized in Table 1. Correlations among BPU, BPE, FGT, and age for both interpreters are summarized in Figure 1. Correlation coefficients ranged from moderate, that is, 0.3-0.5 (between BPU and age, BPE and FGT, and BPE and FGT) to strong, that is, 0.5-0.7 (between age and BPU, BPE, and FGT).

BPU showed a significant moderate direct correlation with BPE $(P<0.001, \rho=0.492)$, indicating that patients with greater levels

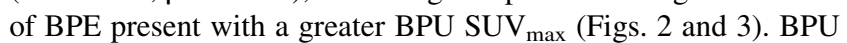
showed a significant moderate direct correlation with FGT $(P<$ $0.001, \rho=0.370)$, indicating that patients with a larger amount of

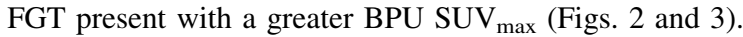

All 3 imaging-derived tissue biomarkers, that is, BPU $(r=0.386)$, BPE $(\rho=-0.498)$, and FGT $(\rho=-0.631)$, showed a significant indirect correlation with age $(P<0.001)$.

When moderate and marked BPE were grouped together, the results were not different. Mean BPU for the grouping was $2.25 \pm$ 0.6. The correlations between BPU, BPE, FGT, and age for this grouped analysis for both interpreters are summarized in Supplemental Figure 1 (available at http://jnm.snmjournals.org). The correlation coefficients ranged from moderate $(0.3-0.5$, between BPE and FGT) to strong (0.5-0.7, between age and BPE). BPU showed a significant moderate direct correlation with $\operatorname{BPE}(P<0.001, \rho=$ $0.422)$. FGT showed a significant moderate direct correlation with BPE $(P<0.001, \rho=0.474)$. BPE showed a significant indirect correlation with age $(P<0.001, \rho=-0.498)$.

The coefficient of variance for quantitative BPU measurements with $\mathrm{SUV}_{\max }$ was $5.6 \%$, indicating a high reproducibility. Interand intrainterpreter agreement for BPE was almost perfect, with $\kappa$-values of 0.860 and 0.822 , respectively. Inter- and intrainterpreter agreement for FGT was also almost perfect, with к-values of 0.839 and 0.931 , respectively.

\section{DISCUSSION}

PET imaging has been implemented worldwide in oncologic practice and is used in breast assessment with promising results $(19,22,29,30)$. BPE and FGT on MRI have been implicated as sensitive predictive and prognostic imaging biomarkers in breast cancer $(12-15,17,31)$. The investigation of BPU as another potential sensitive and also quantifiable imaging biomarker for breast cancer prediction, prognosis, and risk assessment is, therefore, of considerable interest. To fully elucidate the value of BPU, BPE, and FGT as imaging biomarkers for breast cancer, information about their correlations and reproducibility is needed.

The results of our study demonstrate that BPU, which is the degree of ${ }^{18} \mathrm{~F}$-FDG uptake in the glandular tissues of the normal breast, varies among patients. BPU was directly correlated with both MRI-derived BPE and FGT. Patient age showed a moderate to strong indirect correlation with all 3 imaging-derived tissue biomarkers. BPU measurements were highly reproducible, and there was almost perfect inter- and intrainterpreter agreement for both BPE and FGT with MRI. These findings indicate that BPU may serve as a sensitive predictive and prognostic imaging biomarker for breast cancer prediction, prognosis, and risk assessment.

To our knowledge, this is the first study to examine the correlation of BPU on ${ }^{18}$ F-FDG PET/CT with BPE and FGT on MRI. We identified a significant direct correlation between BPU and BPE and between BPU and FGT, which suggests that BPU could also be used as an imaging biomarker for risk assessment, hormonal replacement therapy, or chemoprevention. Just as can be done with the imaging biomarkers BPE and FGT on MRI, BPU can be assessed noninvasively on ${ }^{18} \mathrm{~F}$-FDG PET/CT, but a definite benefit of BPU over BPE and FGT is that it can be assessed quantitatively and is highly reproducible, which is a prerequisite for a stable and clinically relevant imaging biomarker $(32,33)$.

To our knowledge, the correlation of ${ }^{18}$ F-FDG radiotracer uptake in normal breast parenchyma with BPE has been investigated in only a single retrospective study that used ${ }^{18} \mathrm{~F}-\mathrm{FDG}$ positron emission mammography (PEM) (34). In that study, Koo et al. assessed the correlation of BPU on ${ }^{18} \mathrm{~F}$-FDG PEM, BPE on contrast-enhanced MRI, and breast density on mammography. Although mean BPU

TABLE 1

ACR Classification Results for BPE and FGT

\begin{tabular}{lccc}
\hline Parameter & Interpreter 1 & $\begin{array}{c}\text { Reassessment } \\
\text { by interpreter 1 }\end{array}$ & Interpreter 2 \\
\hline BPE & & & \\
\hline Minimal & 58 & 61 & 60 \\
\hline Mild & 54 & 52 & 53 \\
\hline Moderate & 14 & 11 & 13 \\
\hline Marked & 3 & 5 & 3 \\
\hline FGT & & & \\
\hline ACR A & 33 & 32 & 30 \\
\hline ACR B & 59 & 61 & 63 \\
\hline ACR C & 27 & 24 & 22 \\
\hline ACR D & 10 & 12 & 14 \\
\hline
\end{tabular}




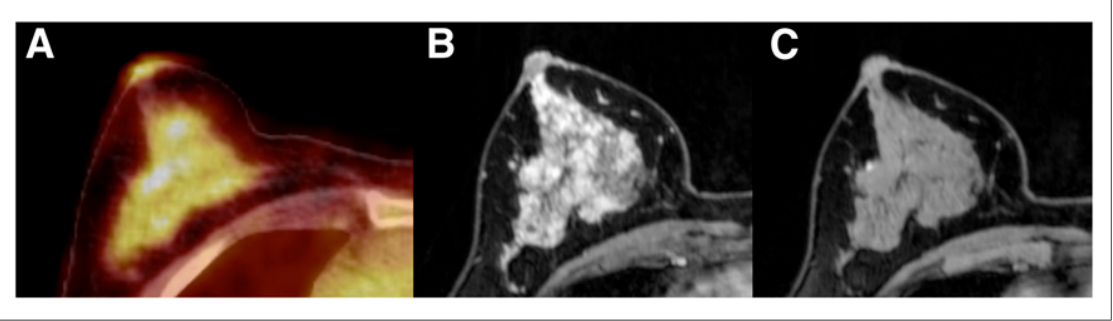

FIGURE 2. 54-y-old woman who presented with BPU SUV $\max$ of 2.96 on ${ }^{18} \mathrm{~F}-\mathrm{FDG}$ PET/CT and showed marked BPE and extreme FGT (ACR D). Axial ${ }^{18} \mathrm{~F}-\mathrm{FDG}$ PET/CT (A), axial dynamic contrastenhanced MRI (B), and axial unenhanced T1-weighted MRI (C) of right breast are shown.

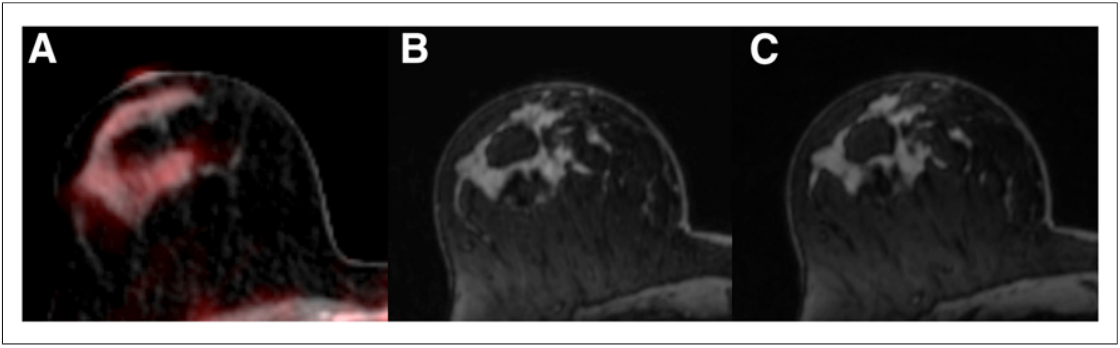

FIGURE 3. 61-y-old woman who presented with BPU SUV max $_{\text {of }} 1.12$ on ${ }^{18} \mathrm{~F}-\mathrm{FDG}$ PET/CT and showed no BPE and scattered FGT (ACR B). Axial ${ }^{18} \mathrm{~F}-\mathrm{FDG}$ PET/CT (A), axial dynamic contrastenhanced MRI (B), and axial unenhanced T1-weighted MRI (C) of right breast are shown.

increased from minimal to marked BPE, there was considerable overlap, especially for women with minimal and mild BPE, and mean BPU was not an independent predictor of BPU on PEM. In contrast, in the current study, we found a direct correlation between BPU and BPE. The divergent results might be explained by the fact that, in the study by Koo et al., there was a small sample size, especially for moderate $(n=5)$ and marked BPE $(n=5)$, which may have led to underpowered statistical analyses. Moreover, in contrast to the current study, BPU was assessed using a 2-dimensional imaging modality, that is, ${ }^{18} \mathrm{~F}-\mathrm{FDG}$ PEM, which could have influenced the results and, thus, limits comparability. In addition, MRI was not scheduled according to the menstrual cycle, and there is no information on the time interval between PEM and MRI, which potentially might act as another confounding factor.

To our knowledge, no information currently exists on the correlation between BPU and FGT on MRI, which is a newly introduced descriptor in the MRI BI-RADS lexicon. In this first study to examine the correlations between BPU and FGT, we found a direct correlation. This finding agrees with studies that have investigated the correlation between BPU on ${ }^{18}$ F-FDG PET or PEM and breast density on mammography, which is the equivalent of FGT on MRI (34-37). These results further underline the potential of BPU as a valuable imaging biomarker in breast cancer.

Findings of increased breast density on mammography have been shown to lessen with age. Similar to mammographic breast density, all 3 tissue-derived imaging biomarkers in our study showed an indirect correlation with patient age. To date, results on the correlation of BPU with age are scarce and divergent. Similar to our results, Mavi et al. (37), Zytoon et al. (29), and Koo et al. (34) demonstrated that ${ }^{18}$ F-FDG uptake significantly decreases as age increases. Only one study, by Vranjesevic et al., found no significant correlation (35).
BPE and FGT also showed a negative correlation with age, as agrees with previously published data from studies that investigated BPE and mammographic breast density (38-40).

A clinically relevant imaging biomarker must be stable and reproducible. In this study, we demonstrated that BPU possesses those qualities, as the quantitative BPU measurements were highly reproducible. Our findings are in accordance with those of Vranjesevic et al. (35) and Mavi et al. (37), who found good agreement between quantitative BPU measurements when determined by 2 observers independently. In our study, there was almost perfect interand intrainterpreter agreement for both BPE and FGT on MRI. Previous studies reported good agreement for BPE classification when performed by trained interpreters $(17,41)$ but yielded more variability for inexperienced interpreters, thus somewhat limiting the stability of BPE as an imaging biomarker. The excellent results in the current study can be explained by the fact that both interpreters were experienced in breast density assessment and MRI. Currently, there are no data on interand intraobserver agreement for FGT, as this parameter has only recently been introduced into the MRI BI-RADS lexicon. In contrast to mammographic breast density (42), for which there is considerable variability, inter- and intrainterpreter agreement was almost perfect for FGT. Similar to the excellent results for BPE, this good agreement is most likely due to the fact that our interpreters had vast experience in breast density assessment and MRI.

The current study has some limitations, one of which is the small number of patients with marked BPE. However, this small number reflects the normal distribution of BPE in the population. Additionally, we performed a grouped analysis for patients with greater levels of BPE - that is, moderate and marked-that yielded similar results. Our study assessed BPE and FGT qualitatively, in contrast to BPU. However, the BI-RADS lexicon does not recommend quantitative assessment of BPE and FGT, but there was excellent intra- and interinterpreter agreement for BPE and FGT. Therefore, the approach in our study seems to be justified. The fact that our study did not perform all PET/CT and MRI examinations on the same day (range, 0-6 d) might have affected BPE in a few cases because of the changes in BPE with the menstrual cycle. However, in most cases, the interval between examinations was short (mean, $2.26 \mathrm{~d}$ ), and thus, relevant changes in BPE seem unlikely.

\section{CONCLUSION}

The results of our study demonstrate that BPU varies among patients. BPU directly correlated with both BPE and FGT on MRI. Patient age showed a strong inverse correlation with all 3 imagingderived tissue biomarkers. BPU measurements were highly reproducible, with almost perfect inter- and intrainterpreter agreement for both BPE and FGT on MRI. These findings indicate that BPU may serve as a sensitive imaging biomarker for breast cancer prediction, prognosis, and risk assessment. 


\section{DISCLOSURE}

The costs of publication of this article were defrayed in part by the payment of page charges. Therefore, and solely to indicate this fact, this article is hereby marked "advertisement" in accordance with 18 USC section 1734 . No potential conflict of interest relevant to this article was reported.

\section{REFERENCES}

1. Lee K, Rossi C. Risk assessment, genetic counseling, and genetic testing for BRCA-related cancer in women. Am Fam Physician. 2015;91:119-120.

2. Lahart IM, Metsios GS, Nevill AM, Carmichael AR. Physical activity, risk of death and recurrence in breast cancer survivors: a systematic review and metaanalysis of epidemiological studies. Acta Oncol. 2015;54:635-654.

3. Morrow M, Jordan VC. Tamoxifen for the prevention of breast cancer in the high-risk woman. Ann Surg Oncol. 2000;7:67-71.

4. Cuzick J, Forbes JF, Sestak I, et al. Long-term results of tamoxifen prophylaxis for breast cancer: 96-month follow-up of the randomized IBIS-I trial. J Natl Cancer Inst. 2007;99:272-282.

5. Burke EE, Portschy PR, Tuttle TM. Prophylactic mastectomy: who needs it, when and why. J Surg Oncol. 2015;111:91-95.

6. Advani P, Moreno-Aspitia A. Current strategies for the prevention of breast cancer. Breast Cancer (Dove Med Press). 2014;6:59-71.

7. Lostumbo L, Carbine NE, Wallace J. Prophylactic mastectomy for the prevention of breast cancer. Cochrane Database Syst Rev. 2010;CD002748.

8. Kinsinger LS, Harris R, Woolf SH, Sox HC, Lohr KN. Chemoprevention of breast cancer: a summary of the evidence for the U.S. Preventive Services Task Force. Ann Intern Med. 2002;137:59-69.

9. Parker WH. Bilateral oophorectomy versus ovarian conservation: effects on long-term women's health. J Minim Invasive Gynecol. 2010;17:161-166.

10. Brandberg Y, Arver B, Johansson H, Wickman M, Sandelin K, Liljegren A. Less correspondence between expectations before and cosmetic results after risk-reducing mastectomy in women who are mutation carriers: a prospective study. Eur J Surg Oncol. 2012;38:38-43.

11. Morris JL, Gordon OK. Positive Results: Making the Best Decisions When You're at High Risk for Breast or Ovarian Cancer. Amherst, NY: Prometheus Books; 2010:89-112.

12. King V, Brooks JD, Bernstein JL, Reiner AS, Pike MC, Morris EA. Background parenchymal enhancement at breast MR imaging and breast cancer risk. Radiology. 2011;260:50-60.

13. Dontchos BN, Rahbar H, Partridge SC, et al. Are qualitative assessments of background parenchymal enhancement, amount of fibroglandular tissue on MR images, and mammographic density associated with breast cancer risk? Radiology. 2015;276:371-380.

14. Kim MY, Cho N, Koo HR, et al. Predicting local recurrence following breastconserving treatment: parenchymal signal enhancement ratio (SER) around the tumor on preoperative MRI. Acta Radiol. 2013;54:731-738.

15. Kim SA, Cho N, Ryu EB, et al. Background parenchymal signal enhancement ratio at preoperative MR imaging: association with subsequent local recurrence in patients with ductal carcinoma in situ after breast conservation surgery. $R a$ diology. 2014;270:699-707.

16. McCormack VA, dos Santos Silva I. Breast density and parenchymal patterns as markers of breast cancer risk: a meta-analysis. Cancer Epidemiol Biomarkers Prev. 2006;15:1159-1169.

17. Preibsch H, Wanner L, Bahrs SD, et al. Background parenchymal enhancement in breast MRI before and after neoadjuvant chemotherapy: correlation with tumour response. Eur Radiol. 2016;26:1590-1596.

18. Wengert GJ, Helbich TH, Vogl WD, et al. Introduction of an automated userindependent quantitative volumetric magnetic resonance imaging breast density measurement system using the Dixon sequence: comparison with mammographic breast density assessment. Invest Radiol. 2015;50:73-80.

19. Pinker K, Bogner W, Baltzer P, et al. Improved differentiation of benign and malignant breast tumors with multiparametric ${ }^{18}$ fluorodeoxyglucose positron emission tomography magnetic resonance imaging: a feasibility study. Clin Cancer Res. 2014;20:3540-3549.

20. Avril N, Adler LP. F-18 fluorodeoxyglucose-positron emission tomography imaging for primary breast cancer and loco-regional staging. Radiol Clin North Am. 2007;45:645-657.

21. Miles KA, Williams RE. Warburg revisited: imaging tumour blood flow and metabolism. Cancer Imaging. 2008;8:81-86.

22. Magometschnigg HF, Baltzer PA, Fueger B, et al. Diagnostic accuracy of ${ }^{18}$ F-FDG PET/CT compared with that of contrast-enhanced MRI of the breast at 3 T. Eur J Nucl Med Mol Imaging. 2015;42:1656-1665.

23. Koolen BB, van der Leij F, Vogel WV, et al. Accuracy of ${ }^{18}$ F-FDG PET/CT for primary tumor visualization and staging in T1 breast cancer. Acta Oncol. 2014;53:50-57.

24. Moy L, Noz ME, Maguire GQ Jr, et al. Prone mammoPET acquisition improves the ability to fuse MRI and PET breast scans. Clin Nucl Med. 2007;32:194-198.

25. Koolen BB, Vidal-Sicart S, Benlloch Baviera JM, Valdes Olmos RA. Evaluating heterogeneity of primary tumor ${ }^{18} \mathrm{~F}$-FDG uptake in breast cancer with a dedicated breast PET (MAMMI): a feasibility study based on correlation with PET/CT. Nucl Med Commun. 2014;35:446-452.

26. Fraum TJ, Fowler KJ, McConathy J. PET/MRI: emerging clinical applications in oncology. Acad Radiol. 2016;23:220-236.

27. Mann RM, Balleyguier C, Baltzer PA, et al. Breast MRI: EUSOBI recommendations for women's information. Eur Radiol. 2015;25:3669-3678.

28. BI-RADS Atlas. 5th ed. Reston, VA: American College of Radiology; 2013.

29. Zytoon AA, Murakami K, El-Kholy MR, El-Shorbagy E. Dual time point FDGPET/CT imaging...potential tool for diagnosis of breast cancer. Clin Radiol. 2008;63:1213-1227.

30. Kalles V, Zografos GC, Provatopoulou X, Koulocheri D, Gounaris A. The current status of positron emission mammography in breast cancer diagnosis. Breast Cancer. 2013;20:123-130.

31. Park VY, Kim EK, Kim MJ, Yoon JH, Moon HJ. Breast parenchymal signal enhancement ratio at preoperative magnetic resonance imaging: association with early recurrence in triple-negative breast cancer patients. Acta Radiol. 2016;57:802-808.

32. Prescott JW. Quantitative imaging biomarkers: the application of advanced image processing and analysis to clinical and preclinical decision making. J Digit Imaging. 2013;26:97-108.

33. European Society of Radiology. White paper on imaging biomarkers. Insights Imaging. 2010;1:42-45.

34. Koo HR, Moon WK, Chun IK, et al. Background ${ }^{18}$ F-FDG uptake in positron emission mammography (PEM): correlation with mammographic density and background parenchymal enhancement in breast MRI. Eur J Radiol. 2013;82:1738-1742.

35. Vranjesevic D, Schiepers C, Silverman DH, et al. Relationship between ${ }^{18}$ F-FDG uptake and breast density in women with normal breast tissue. J Nucl Med. 2003;44:1238-1242.

36. Kumar R, Chauhan A, Zhuang H, Chandra P, Schnall M, Alavi A. Standardized uptake values of normal breast tissue with 2-deoxy-2-[F-18]fluoro-D-glucose positron emission tomography: variations with age, breast density, and menopausal status. Mol Imaging Biol. 2006;8:355-362.

37. Mavi A, Cermik TF, Urhan M, et al. The effect of age, menopausal state, and breast density on ${ }^{18} \mathrm{~F}$-FDG uptake in normal glandular breast tissue. J Nucl Med. 2010;51:347-352.

38. Müller-Schimpfle M, Ohmenhauser K, Stoll P, Dietz K, Claussen CD. Menstrual cycle and age: influence on parenchymal contrast medium enhancement in MR imaging of the breast. Radiology. 1997;203:145-149.

39. Carney PA, Miglioretti DL, Yankaskas BC, et al. Individual and combined effects of age, breast density, and hormone replacement therapy use on the accuracy of screening mammography. Ann Intern Med. 2003;138:168-175.

40. Kelemen LE, Pankratz VS, Sellers TA, et al. Age-specific trends in mammographic density: the Minnesota Breast Cancer Family Study. Am J Epidemiol. 2008; 167:1027-1036.

41. Melsaether A, McDermott M, Gupta D, Pysarenko K, Shaylor SD, Moy L. Interand intrareader agreement for categorization of background parenchymal enhancement at baseline and after training. AJR. 2014;203:209-215.

42. Ciatto S, Houssami N, Apruzzese A, et al. Categorizing breast mammographic density: intra- and interobserver reproducibility of BI-RADS density categories. Breast. 2005;14:269-275. 